\title{
Abuses in Cryptography and How to Fight Them
}

\author{
(Extended Abstract)
}

\author{
Yvo Desmedt \\ Dept. EE \& CS, Univ. of Wisconsin-Milwaukee \\ P.O. Box 784, WI 53201 Milwaukee, U.S.A.
}

\begin{abstract}
The following seems quite familiar: "Alice and Bob want to fip a coin by telephone. (They have just divorced, live in different countries, want to decide who will have the children during the next holiday.)..." So they use [Blu82]'s (or an improved) protocol. However, Alice and Bob's divorce has been set up to cover up their spying activities. When they use [Blu82]'s protocol, they don't care if the "coinflip" is random, but they want to abuse the protocol to send secret information to each other. The counter-espionage service, however, doesn't know that the divorce and the use of the [Blu82]'s protocol are just cover-ups.

In this paper, we demonstrate how several modern crypto-systems can be abused. We generalize [Sim83b]'s subliminal channel and [DGB87]'s abuse of the [FFS87, FS86] identification systems and demonstrate how one can prevent abuses of cryptosystems.
\end{abstract}

\section{Introduction}

[Sim83b] introduced the notion of subliminal channel. His example is related to two prisoners who are communicating authenticated messages in full view of a warden, who is able to read the messages. The subliminal consists in hiding a message through the authentication scheme such that the warden cannot detect its use nor read the hidden part. At Crypto'87, [DGB87] discussed a similar scenario by demonstrating that the [FFS87,FS86] identification systems can be abused for sending secret messages in an undetectable way. Claiming that he is identifying himself, [DGB87] enables, for example, a mafia Godfather to communicate under the F.B.I.'s very nose without having to worry that it would be detected. In this paper we will generalize these undetectable abuses and subliminal channels. We prefer to use the term abuse in the general context and reserve the word subliminal in the special context that it is an abuse of an authentication or signature system. We will briefly demonstrate 
that many modern crypto-systems can be abused (see Section 2). Abuses (in particular subliminal channels) are not covert channels in the strict way, as will briefly be discussed in Section 2.2 .

The main purpose of this paper is to make abuse-free crypto-systems (including protocols). In Section 2.3, we will propose the main tool, while in Section 3 we will give general solutions to solve the abuse problem. Specific applications, such as coin flipping over the telephone, and subliminal-free authentication- and signature systems will be focused on in more detail in Section 4 .

\section{Abuses}

\subsection{AN INTRODUCTION}

The problem of fraud, such as eavesdropping and modification of messages, is well known. It can be said that modern cryptography studies the methods used to protect data against several types of fraud. A crypto-system protects data against a subset of frauds. For example, [BG84] protects the information against an eavesdropper who would use ciphertext-only and known-plaintext, but not chosen ciphertext attacks.

Let us now discuss what an abuse is. In order for our definition to make sense, we need a warden, as in [Sim83b]. If $A$ uses a crypto-system or is a party of a protocol, we say that $A$ can abuse the system if she is able to use it for a different purpose than for which it is intended. An abuse is undetectable if it is impossible for the warden to detect (in polynomial time) that $A$ uses the abuse. It is trivial to make detectable abuses. So we will only discuss undetectable abuses and will call them briefly: "abuses". A particular abuse is that $A$ is able to send (encrypted) information to other parties involved besides the warden.

A formal and more general definition of an abuse can be given (see [Des]), but this formal definition is complex and therefore not covered here. Informally, if $A$ is supposed to use a crypto-system $C$ (or is a party of a cryptographic protocol), but uses a different special system $\left(C^{\prime}\right)$, we say that $A$ can abuse the system if the numbers that she sends:

- do not allow the warden to distinguish (in polynomial time) between normal use and special use,

- allow a participant (e.g. $B$ ) to distinguish with high enough probability.

It is trivial to understand that an abuse can consist in replacing the random which is used in a crypto-system by pseudo-nandom, or even by the output of a one-time pad. The user who abuses a system can find the use of his abuse more important than endangering the security of the system, in particular his secret. Abusing systems as: [Blu82], and zero-knowledge is trivial and it is remarkable that this aspect 
of crypto-systems has never been studied before beside in a narrow context as authentication [Sim83b] and identification [DGB87]. Remark that the goal of an abuse can be considered as the opposite of the goal of zero-knowledge.

\subsection{ABUSES VERSUS COVERT CHANNELS}

It is important to remark that, strictly speaking, abuses are not covert channels. According to [Lam73, p. 614]:

Covert channels, i.e. those not intended for information transfer at all, such as the service program's effect on the system load.

A more general definition can be found in [Dep83, p. 110].

It is very important to observe that the Lampson definition implies that abuses are not covert channels. Indeed, messages are transmitted in crypto-systems so they are intended for information transfer. For example, a zero-knowledge protocol intends a very small information transfer.

In this paper we will only discuss abuses and not covert channels. Leaking information through methods such as time jitter, crosstalk and amplitude modulation, as discussed in [Sim88, p. 626], are covert channels, and thus not a topic of this paper.

Whether our solutions against abuses can be extended to covert channels is a new, open question. The author admits that the difference between abuses and covert channels is debatable, and that one could claim that abuses are very special covert channels. In this paper we consider them to be different. What makes abuses so unique is that the hidden information is a "number", and that one can hide it by using a crypto-system. Formalizing the definition of covert channels could imply that the new open problem can be solved.

\subsection{ABUSE-FREENESS}

We will say that a warden is passive if he is just observing the communication between the participants. In the narrow context of authentication, which was studied in [Sim83b], the warden was also passive. Our main solution against abuses is based on an active warden $W$ who does not only listen to catch up subliminal senders, but also interacts in the communication in a special way to better enforce the subliminalfreeness. In other words, he participates actively in the communication between all participants and he can modify the "numbers" that are communicated. The only trust in the active warden consists in believing he will not help to set-up an abuse. One could compare the active wardens with Simmons' idea used to exclude the use of analog covert channels [Sim83a, p. 65]. The main difference is that the active warden is digital.

Informally, we said that a system can be abused if another system exists such 
that the warden cannot distinguish between normal execution and the execution of the other system, while a participant can. So, abuse-freeness can be considered as the logical negation of the existence of an abuse. It means: if a participant is able to distinguish between execution of the normal system and a different one, then the warden can distinguish it also, as well as for all possible different systems, which are different from the normal system. Hereto the warden will modify the numbers that are transmitted. An exact and more formal definition is given in [Des], but not included here because it is too lengthy and complicated.

Evidently, the action of the warden may not endanger the security of the system.

\subsection{Practical aspects of the WARdeN}

One could wonder if it is possible to construct abuse-free crypto-systems where the warden is passive. In Section 3.3 we will briefly discuss this topic.

In our solution, one assumes that there is one (active) warden. In some situations, as in the verification of treaties [Ada88,Sim88], it is in reality sufficient and achievable to have only one active warden. However, in some circumstances having only one warden is insecure or impractical. [Des] discusses these situations and proposes better models. Let us briefly overview them.

Goutier [DGB87] remarked that the subliminal sender can also send information to others who are eavesdropping on the communications. Indeed, passive and active eavesdroppers can be subliminal receivers. In this paper, we assume that this isn't the case. It is not hard to generalize our results in order to solve the problem of eavesdroppers who are subliminal receivers by using two wardens to protect a communication link, one at each end of the line. These two wardens could trust or distrust each other. Some of our protocols can easily be adapted to it. Other problems are:

- that we assume that the warden himself will not try to abuse the system; implying that we trust the warden will not try to send hidden information,

- speed and number of interactions between warden and participants,

- that it is not excluded that participants have been able to hide a covert or physical channel with small capacity. The warden is unaware of this extra channel. The existence of this hidden channel could imply that the system, which was originally abuse-free, is no more due to this extra information.

We call this last problem the collapse problem. These topics and how to solve them are discussed in [Des].

We now discuss how the idea of an active and censoring warden can actually be used. The main techniques that we use are: commitment, zero-knowledge [GMR], and the one-time pad crypto-system. 
We will start by discussing the more general cases first. Proofs will not be given because they require a formal definition.

\section{Abuse-free systems in a general context}

Zero-knowledge allows $A$ to restrict leaks of information if $A$ wants. We demonstrate that a warden can enforce $A$ not to leak information, even if $A$ tries her hardest. Hereto, we first discuss in Section 3.1, in general terms, how to generate a public key in an abuse-free way. We then discuss how to make interactive and non-interactive zero-knowledge abuse-free.

\subsection{How TO GENERATE A PUBLIC KEY IN AN ABUSE-FREE WAY}

Motivation

Publishing a public key can be abused. To illustrate, suppose that $A$ publishes a public key $n=p q$, where $p$ and $q$ are primes of 100 digits. If $A$ is able and/or allowed to give $B$ a 100 digit number e.g., $p$, it is trivial to understand that, by publishing $n$, $A$ is able to leak 100 digits of extra information to $B$ (for improvements see [RS85]). Another method for leaking information is to choose $p$ and $q$ such that the least significant bits of $n$ have a special form not required by the specifications.

So the process of publishing a key is abuse-free if the key is guaranteed to be random beside the specifications e.g., a product of two large different primes both congruent to 3 modulo 4 .

A solution could be that the warden chooses the public key of $A$. However, this allows the warden to become Big Brother. We exclude this solution.

\section{A solution}

To generate the public key, $A$ normally chooses some random number, $R$ and verifies if $R$ satisfies conditions, $C$ and if so calculates public key, $P=\operatorname{GEN}(R)$, where GEN is a publicly known algorithm. However it is also possible that $R$ is not suited (does not satisfy $C$ ) e.g., $p$ and $q$ that are composite numbers are unsuited for RSA. In the last case, we require that $A$ must be able to convince the active warden, $W$ that this $R$ is unsuited to make $P$. Roughly speaking, to obtain an abuse-free public key, $A$ will use her own generated random $(R)$ exored with random $\left(R^{\prime}\right)$ generated by $W$, to make the public key $P$. The following protocol makes it clear that no cheating is possible.

First $W$ and $A$ agree on a commitment algorithm (or circuit) $E$, such that the commitment can be verified in random polynomial time. They also agree on algorithm TESTC to verify that $R$ satisfies conditions $C$, and on algorithm TESTNOC to verify that $R$ does not. We then have: 
Step $1 A$ chooses a (random) binary string $R=\left(r_{1}, r_{2}, \ldots, r_{l}\right)$ of $l$ bits, an appropriate $k$, and $A$ sends $M=E_{k}(R)$ to $W$ as a commitment for $R$.

Step $2 W$ chooses a truly random binary string $R^{\prime}=\left(r_{1}^{\prime}, r_{2}^{\prime}, \ldots, r_{l}^{\prime}\right)$ of $l$ bits and sends it to $A$.

Step $3 A$ calculates $S=\left(r_{1} \oplus r_{1}^{\prime}, r_{2} \oplus r_{2}^{\prime}, \ldots, r_{l} \oplus r_{l}^{\prime}\right)$ ( $\oplus$ is exclusive or). Shortly we denote $S=R \oplus R^{\prime}$. If $S$ satisfies conditions $C$ (case 1) then $A$ calculates $P=\operatorname{GEN}(S)$ and sends $P$ to $W . A$ then proves to $W$ that there exists $R=\left(r_{1}, r_{2}, \ldots, r_{l}\right)$ and $k$ such that:

$$
M=E_{k}(R) \wedge \quad \operatorname{TESTC}\left(R \oplus R^{\prime}\right) \quad \wedge \quad P=\operatorname{GEN}\left(R \oplus R^{\prime}\right) .
$$

This proof has to be zero-knowledge. Else (case 2) $A$ convinces $W$ that $S$ does not satisfy conditions $C$. In this case, $A$ can even reveal $R$ and $k$ to convince $W$.

Step $4 W$ verifies $A$ 's proof. If this proof fails, $W$ stops protocol, else one continues. In the case that a $P$ was delivered by $A$ (case 1 ), $W$ publishes $A$ 's public key $P$ and protocol halts. Else (in case 2) the protocol restarts from the beginning (Step 1).

\section{Important remarks}

Security (privacy of the secret key) and abuse-freeness of this protocol are proven in [Des]. The security is based on the assumption that the commitment algorithm is hard to invert. Indeed, if the commitment algorithm could be broken, the warden will know $A$ 's secret key. The abuse-freeness is unconditional.

In most cases the zero-knowledge proof which is given in Step 3 is impractical and too slow, certainly when it has to be based on [GMW86]. But because public keys are only generated occasionally, this is of less importance. If we would use [BC86], the unconditionality of the abuse-freeness disappears.

\subsection{ABUSE-FREE INTERACTIVE ZERO-KNOWLEDGE}

Many practical zero-knowledge protocols can be made abuse-free. We will mainly give a general result by demonstrating how to make the [GMW86] zero-knowledge proof of 3-colourability abuse-free, and indicate how one can make the [BC86] zeroknowledge proof for SAT abuse-free. Let us start with [GMW86].

We use the same notation as in [GMW86, pp.176-177], but we number the edges as $E=\{0,1, \ldots, m-1\}$ and we call the prover, $A$ and the verifier, $B$. One agrees that the protocol will always end after $l$ iterations, $l$ in function of the (security) parameters. The main problem is that $A$ will reveal $\left(\pi(\phi(u)), r_{u}\right)$ and $\left(\pi(\phi(v)), r_{v}\right)$, which could be abused. A similar approach as in Section 3.1 could be followed but would be very impractical. The following protocols avoid this. It is organized 
such that all numbers that are sent cannot be abused. The warden will influence all numbers that are transmitted from $A$ to $B$ and vice-versa.

Step $1 A$ chooses a (random) permutation $\pi \in S y m(\{1,2,3\})$ and (random) $r_{v}, r_{v}^{\prime}$ and $k_{v}$ (large enough) and computes the commitments $R_{v}=$ $f\left(\pi(\phi(v)), r_{v}\right)$ and $K_{v}=f\left(k_{v}, r_{v}^{\prime}\right)$ (for all $v \in V$ ), and sends $R_{1}, R_{2}$, $\ldots, R_{n}$ and $K_{1}, K_{2}, \ldots, K_{n}$ to $W$.

Step $2 W$ chooses a truly random $\pi^{\prime} \in_{R} \operatorname{Sym}(\{1,2,3\})$ and truly random $k_{1}^{\prime}, k_{2}^{\prime}$, $\ldots, k_{n}^{\prime}$ and sends them to $A$.

Step $3 A$ calculates $\pi^{\prime \prime}=\pi^{\prime} \pi$ and $r_{v}^{\prime \prime}=k_{v} \oplus k_{v}^{\prime}$ and $R_{v}^{\prime}=f\left(\pi^{\prime \prime}(\phi(v)), r_{v}^{\prime \prime}\right.$ ) (for all $v \in V$ ) and sends $R_{1}^{\prime}, R_{2}^{\prime}, \ldots, R_{n}^{\prime}$ to $W$.

Step $4 \mathrm{~W}$ chooses truly random $s_{v}$ and calculates $R_{v}^{\prime \prime}=f\left(R_{v}^{\prime}, s_{v}\right)$ (for all $v \in V$ ) and sends $R_{1}^{\prime \prime}, R_{2}^{\prime \prime}, \ldots, R_{n}^{\prime \prime}$ to $B$.

Step $5 B$ selects (at random) an edge $e \in E$ and a (random) $t$ and sends $S=$ $f(e, t)$ to $W$.

Step $6 W$ chooses a truly random $e^{\prime} \in_{R} E$ and sends it $B$.

Step $7 B$ reveals $e$ and $t$ to $W$.

Step $8 W$ verifies if $e \in E$ and checks whether $S=f(e, t)$. If both conditions are satisfied, then $W$ calculates $e^{\prime \prime}=e+e^{\prime} \bmod m$ (edges where hereto specially numbered) and sends $e^{\prime \prime}$ to $A$. Else $W$ stops protocol.

Step 9 Let $(u, v)$ correspond with $e^{\prime \prime}$, where $u, v \in V$ and $u<v$. A reveals $\left(\pi^{\prime \prime}(\phi(u)), r_{u}^{\prime \prime}, r_{u}^{\prime}, r_{u}\right)$ and $\left(\pi^{\prime \prime}(\phi(v)), r_{v}^{\prime \prime}, r_{v}^{\prime}, r_{v}\right)$ to $W$. If $e^{\prime \prime} \notin E$ (W cheats), then $A$ stops.

Step $10 W$ uses the information revealed by $A$ to check $R_{u}^{\prime}$. Then $W$ calculates $k_{u}$ $\left(k_{u}=r_{u}^{\prime \prime} \oplus k_{u}^{\prime}\right)$ and verifies $K_{u}$. He then calculates $\pi(\phi(u))$ (starting from $\pi^{\prime \prime}(\phi(u))$ and $\left.\pi^{\prime}\right)$ and verifies $R_{u}$. He does exactly the same to verify $R_{v}^{\prime}$, $K_{v}$ and $R_{v}$. W then checks if $\pi(\phi(u)) \neq \pi(\phi(v))$ and $\pi(\phi(u)), \pi(\phi(v)) \in$ $\{1,2,3\}$. If either condition is violated, $W$ rejects and stops protocol, else $W$ reveals $\left(\pi^{\prime \prime}(\phi(u)), r_{u}^{\prime \prime}, s_{u}\right)$ and $\left(\pi^{\prime \prime}(\phi(v)), r_{v}^{\prime \prime}, s_{v}\right)$ to $B$.

Step $11 B$ checks if $R_{u}^{\prime \prime}=f\left(f\left(\pi^{\prime \prime}(\phi(u)), r_{u}^{\prime \prime}\right), s_{u}\right)$ and similar for $R_{v}^{\prime \prime}$. He also checks if $\pi^{\prime \prime}(\phi(u)) \neq \pi^{\prime \prime}(\phi(v))$ and $\pi^{\prime \prime}(\phi(u)), \pi^{\prime \prime}(\phi(v)) \in\{1,2,3\}$. If either condition is violated, $B$ rejects and stops protocol, else one continues with the next iteration if the number of iterations is less than $l$ (else stops and $B$ accepts).

As in [GMW86], $\phi$ is never released. Their main theorems remain valid, so abusefree zero-knowledge protocols exist for all NP languages. The abuse-freeness of the above protocol is not unconditional. 
If $W$ is forced to stop the protocol (see Step 8 and Step 10), one could correctly remark that $A$ or $B$ has succeeded in leaking one bit of information. However, this is not an abuse according to our definition because the warden can detect it, too. In practice it means that one is able to leak one bit of information (the fact that $W$ was forced to stop the protocol), however, the risk to be caught is too high to attempt it. The same remark is valid for most protocols that we will discuss further in this paper. We will not repeat this remark.

Let us now explain how to make [BC86] zero-knowledge proof abuse-free. We will use the same notations as in [BC86]. Here, we only demonstrate how $A$ can prove, in an abuse-free way, to $B$ that $b_{1}=b_{2}$ without revealing them. It is then trivial (see [Des]) to extend the results to make the [BC86] zero-knowledge proofs abuse-free. We assume that $B$ has published abuse-free $y \in \mathrm{QR}_{n}$ and $n$, such that $n=p q$ where $p$ and $q$ are both primes congruent to 3 modulo 4 and convinced $A$ by using a [GMW86] type abuse-free zero-knowledge protocol that $y$ and $n$ satisfy the conditions. Remark that the requirement that $y, n$ and the last proof have to be abuse-freeness can be relaxed. This means, for example, that if $A$ would know the factorization of $n$, it would not help him to abuse the following protocol. The abuse-free protocol to prove that $b_{1}=b_{2}$ is as follows:

Step $1 A$ chooses a (random) $w_{1}$, such that $\operatorname{gcd}\left(w_{1}, n\right)=1$ and calculates $z_{1}=$ $\pm w_{1}^{2} y^{b_{1}} \bmod n . A$ calculates $z_{2}$ in a similar way. $A$ then calculates $w$ as in [BC86] (if $b_{1}=1$ and $b_{2}=1$ then $w=w_{1} w_{2} y \bmod n$, else $w=w_{1} w_{2} \bmod$ $n$ ). $A$ sends $z_{1}, z_{2}$ and $w$ to $W$.

Step $2 W$ verifies if $z_{1} z_{2}= \pm w^{2} \bmod n$ or if $z_{1} z_{2}= \pm w^{2} y \bmod n$. He also verifies if the Jacobi symbols $\left(z_{1} \mid n\right)=1$ and $\left(z_{2} \mid n\right)=1$. If either condition is violated $W$ stops protocol, else he chooses truly random $\psi_{1}$ and $\psi_{2}$ coprime with $n$ and sends $z_{1}^{\prime}= \pm \psi_{1}^{2} z_{1} \bmod n, z_{2}^{\prime}= \pm \psi_{2}^{2} z_{2} \bmod n$ and $\omega=\psi_{1} \psi_{2} w \bmod n$ to $B$.

Step $3 B$ verifies if $z=z_{1}^{\prime} z_{2}^{\prime}= \pm \omega^{2} \bmod n$, then $b_{1}=b_{2}$, else $b_{1} \neq b_{2}$.

The abuse-freeness is unconditional and the protocol is practical. Remark that in the original [BC86] proof, $n$ didn't have to have the special form we request, and the \pm were not used in the protocol. Without these modifications it would have been impossible to make the protocol abuse-free without increasing the overhead enormously. Purdy made the observation to the author that the test of the Jacobi symbol can be eliminated by choosing $p \equiv 3 \bmod 8$ and $q \equiv 7 \bmod 8$. However, it must then be replaced by a test for ged, which is almost as involved.

\subsection{AbUSE-FREE NON-INTERACTIVE ZERO-KNOWLEDGE}

Non-interactive zero-knowledge protocols were introduced by [BFM88]. Let us briefly discuss, from our point of view, the main ideas used in it. Prover and verifier share 
a common random string (the rand tables). The verifier does not need to toss secret coins, however, the prover tosses secret coins. When $A$ proves a theorem, we say that she feeds here private random coin tosses into the proof mechanism.

The main problem from our viewpoint is that nothing guarantees that the prover will indeed toss coins and will not proceed differently in order to abuse. We now sketch how one can make abuse-free non-interactive zero-knowledge (more details are in [Des]).

In the set-up process each individual makes a secret abuse-free seed, in a similar way as in Section 3.1. This means that the individual, $A$, chooses a number, $R$, and that she sends the warden $W$ a commitment to $R$. The warden, $W$, chooses a random number $R^{\prime}$ and sends it to $A$. We call $S=R \oplus R^{\prime}$ (bit by bit exclusive or) the abuse-free seed. Let us now discuss how to proceed when $A$ wants to prove a theorem $\tau$ in non-interactive zero-knowledge. $A$ uses a commonly agreed upon pseudo-noise generator which starts from the abuse-free seed. The output of that generator is fed to the proof mechanism. To understand the idea, it is important to observe that by knowing the seed, the proof mechanism is a deterministic process. $A$ now also generates a non-interactive proof to demonstrate that she used the seed $S$ in the correct way and that indeed $S=R \oplus R^{\prime}$, where the $R^{\prime}$ had been chosen earlier by the warden and that she committed herself to $R$. $R$ itself will never be released! So the warden receives two non-interactive zero-knowledge proofs: the first for theorem $\tau$ and the second to prove that $A$ "decently behaved" when she was proving the first theorem. The warden verifies both theorems and will censor the second one. If both are correct, the warden publishes (or sends) the first proof.

The abuse-freeness is not unconditional. A problem of the above solution is that it suffers from the collapse problem. Indeed, suppose that there exists for a few days a covert channel with small capacity between the prover and a verifier, which cannot be controlled by the warden, $W$. If the prover sends to that verifier the seed $S$ (secret previously unknown), then the zero-knowledge disappears from a practical point of view (it is still theoretically zero-knowledge). The prover can then later abuse the non-interactive protocol to send the complete proof to the verifier and the warden will believe falsely that the verifier will not learn more than the fact to be convinced that the prover knows a proof. The protocols discussed earlier in this paper didn't suffer from this collapse problem.

The warden in this scenario is less active than in previous ones. His only action is verification and censoring, we therefore call him: censoring warden. The idea of censoring warden opens the question if it is possible to reduce the warden's role to a passive one keeping the abuse-freeness. If it would be possible to generate true randomness and to prove in some zero-knowledge way that indeed the numbers are truly random, then the above open problem could be solved. In this context, one could think to use [GMW86, p. 182] ideas to prove pseudorandomness, however in many of the systems discussed here the abuse-freeness is unconditional. So the question 
is if one can benefit from both unconditional abuse-freeness and a passive warden. Making the warden less active does not necessarily imply that the system becomes more practical. Indeed, the above solution is, for the moment, completely impractical because the prover has to perform a tremendous amount of work.

\section{Abuse-free crypto-systems: in narrow contexts}

We will briefly discuss particular abuse-free crypto-systems which are more useful for daily life applications.

\subsection{ABUSE-FREE PRIVACY}

It is possible to make probabilistic public-key encryption systems as [BG84] abusefree. This may seem meaningless. However, it could be that a warden allows $A$ to send $m$ encrypted bits but no more. This can be achieved by making it abuse-free, regardless of the fact that [BG84] expands the data.

\subsection{ABUSE-FREE AUthentication AND SignatURES}

Based on [GMR88], zero-knowledge and the idea of an active warden, a "practical" abuse-free (public-key) authentication system and a less practical abuse-free signature system were presented by the author in [Des88]. The author, [Des88] observed also that in the case that zero-knowledge is combined with [GMR88] for authentication purposes, the authentication tree can be dropped without endangering the proven secure aspect of the scheme. We now discuss a more practical abuse-free signature system.

We briefly discuss here how one can make an abuse-free signature system based on [FFS87,FS86]. Let $\left(n, I_{1}, \ldots, I_{k}\right)$ be an abuse-free public key, such that $n=p q$, where both $p$ and $q$ are congruent to 3 modulo 4 and $I_{i}=s_{i}^{2} \bmod n$. We assume that $n$ has indeed this form and that the warden has been convinced of this for once and for all. When $A$ wants to sign the message $m$ :

Step $1 W$ chooses truly random $r_{i} \in \mathrm{QR}_{n}$, and random $k_{i}$ and sends $E_{k_{i}}\left(r_{i}\right)$ $(1 \leq i \leq t)$ as commitments to $A$.

Step $2 A$ picks (random) $\rho_{i} \in Q R_{n}$ and computes $x_{i}=\rho_{i}^{2} \bmod n$ and sends these $x_{i}$ to $W$.

Step $3 W$ reveals $\left(r_{i}, k_{i}\right)$ to $A$.

Step $4 A$ verifies commitment. If satisfied, $A$ computes $x_{i}^{\prime}=r_{i}^{2} x_{i}$ and computes $e_{i j}$ starting from $x_{i}^{\prime}$, in a similar way as in [FS86].

Step 5 One continues in a similar way as in [FS86] with the $x_{i}^{\prime}$. W verifies if $A$ 
has used the $r_{i}$. A has to prove (using zero-knowledge) to $W$ that all the numbers that she has sent, except the $e_{i j}$, are quadratic residues mod $n$. Then $W$ publishes the signature.

The problem that $f$ is non-random remains, which implies that problems arise to prove the security of the signature system, similarly as in [FS86]. Two aspects of the above protocol can be improved by modifying it. The above protocol is not unconditionally abuse-free. Indeed if the commitment function $E$ can be broken, then $A$ can abuse it. This evil can be overcome by changing the protocol so that $A$ is committing herself, instead of the warden, similarly as it was in all previous protocols. In Step 5, it was mentioned that $A$ has to prove that all numbers (except $e_{i j}$ ) are quadratic residues, this includes all $\rho_{i}$. One can drop the zero-knowledge proof for $\rho_{i}$ if $\rho_{i} \in Z_{n}^{+1}=\left\{y \in Z_{n}^{*} \mid(y \mid n)=1\right\}$, and if the warden can choose randomly, with uniform distribution, in polynomial time $y$ 's with Jacobi symbols $(y \mid n)=1$. Details of the modified algorithm are described in [Des].

\subsection{ABUSE-FREE COIN FLIPPING OVER THE TELEPHONE}

We now apply our tools to make a modified version of Blum's protocol abuse-free.

We will base our solution on this assumption: it is hard to determine if a number is a quadratic residue $\bmod n$. If Alice $(A)$ and $\operatorname{Bob}(B)$ want to flip a coin, then the following protocol is abuse-free:

Step $1 A$ (with $W$ ) generates an abuse-free public key $n$, which is the product of two large distinct primes both congruent to 3 modulo 4 , using the protocol of Section 3.1.

Step $2 A$ generates a (random) $X$, such that $\operatorname{gcd}(X, n)=1$ and sends $Y=$ $\pm X^{2} \bmod n$ to $W$.

Step $3 W$ checks if the Jacobi symbol $(Y \mid n)=1$. If it is not 1 , then $W$ stops protocol (or asks another $Y$ ), else $W$ generates truly random $X^{\prime}$ and \pm 1 , such that $\operatorname{gcd}\left(X^{\prime}, n\right)=1$ and sends $Y^{\prime \prime}= \pm Y X^{\prime 2} \bmod n$ to $B$. If the warden's \pm 1 is 1 , then $g^{\prime}=1$, else $g^{\prime}=0$.

Step $4 B$ guesses if $Y^{\prime \prime}$ is a quadratic residue $\bmod n$. If he thinks it is, then he sends $g=1$ to $W$, else he sends $g=0$.

Step $5 W$ sends $g^{\prime \prime}=g \oplus g^{\prime}$ to $A$.

Step $6 A$ calculates the outcome of the protocol $g^{\prime \prime} \oplus q$, where $q=1$ when $Y$ is quadratic residue $(\bmod n)$, otherwise $q=0 . A$ then reveals $X$ to $W$.

Step $7 W$ verifies $Y$. If it is correct, then $W$ reveals $X^{\prime \prime}=X \cdot X^{\prime} \bmod n$ to $B$, else $W$ stops protocol. 
Step $8 B$ verifies $Y^{\prime \prime}$. $B$ must still be convinced that $n$ is of the appropriate form. Hereto:

Step $9 A$ (with $W$ ) proves to $B$ that $n$ is of the appropriate form by using an abuse-free zero-knowledge protocol.

Step $10 B$ verifies this abuse-free zero-knowledge protocol. If satisfied, $B$ calculates $g \oplus q^{\prime \prime}$ as the outcome of the protocol, where $q^{\prime \prime}=1$ if $Y^{\prime \prime}$ turns out to be a quadratic residue, else $q^{\prime \prime}=0$. Remark that $A$ and $B$ have the same outcome, in other words $g \oplus q^{\prime \prime}=g^{\prime \prime} \oplus q$.

Notice that $A$ and $B$ are not able to abuse one bit, not even the outcome bit. Even if $W$ collaborates with $A$ (or similarly with $B$ ), $A$ cannot benefit from this collaboration to influence the outcome of the protocol in her favor.

The above protocol is not unconditionally abuse-free. The impossibility that $A$ could abuse the coin-flip is unconditional, but $B$ could do it if it were easy for him to determine whether a number is a quadratic residue. This means that there is a small collapse problem. Indeed, if a hidden channel not under control by the warden, exists for a while between $A$ and $B, A$ could use it to send the factorization of $n$ to $B$. Later (when the hidden channel is no longer), when $A$ would choose her \pm in Step 2 in a way that $B$ could predict, $B$ is able to calculate, from $Y^{\prime \prime}$, what the warden's choice for $g^{\prime}$ was. This allows him to choose his $g$ in such a way that $g^{\prime \prime}=g \oplus g^{\prime}$ contains the subliminal information for $A$. The collapse problem is a direct consequence of the fact that the protocol is not unconditionally abuse-free.

The above protocol is very practical beside the fact that in Step 1 one makes an abuse-free public key $n$. The question, if it is allowed to drop this condition as long as $A$ proves to $W$ that $n$ is of the appropriate form, can be derived from the discussion about the small collapse problem, higher up.

[Blu82] already suggested authenticating all communications. It is possible to come up with a system such that $A$ and $B$ can flip a coin in an abuse-free way and authenticate the coin-flip in an abuse-free way by using our ideas of Section 4.2.

\section{Theoretical and practical consequences: conclusion}

Zero-knowledge allows $A$ to restrict leaks of information if $A$ wants. This paper demonstrates that a warden can enforce $A$ not to leak information, even if $A$ tries her hardest. Solutions were presented in a general context and practical solutions were presented in particular contexts. In some of these practical protocols, the warden's role is minimal and it is mainly a multiplication.

Applications of abuse-free cryptography are in the area of international communications as: international bank transfers, authentication of international messages, and treaty verification [Sim83a, $\operatorname{Sim} 88$ ] (in the context of authentication without privacy). If coin-flipping and similar protocols had been used on a large international 
scale, abuses would have formed a threat for (national) security. The above protocols prevent this danger in such a way that users of the system do not have to trust the warden's integrity. Objections against the use of (public key) cryptography (as in [PK79, p. 344]), in particular against authentication, grounded on the fear that terrorists would be able to communicate encrypted information, now vanishes when abuse-free systems are used. So, it also promotes the commercial use of cryptology.

One can wonder if the above solutions are applicable to covert-channel-free computation and computer-security in general.

Acknowledgements: The author thanks the anonymous referees for encouraging the author to make the definitions more precise and to discuss different models for wardens, which are both included in [Des], and to emphasize the difference between covert channels and subliminal channels. George Purdy called the author's attention to the collapse problem. Russell Impagliazzo suggested the name: censoring warden. The author wishes to thank all those who showed interest for this work, in particular, Manuel Blum, Gilles Brassard, David Chaum, Shimon Even, and Jennifer Seberry.

\section{REFERENCES}

[Ada88] J. A. Adam. Ways to verify the U.S.-Soviet arms pact. IEEE Spectrum, pp. 30-34, February 1988.

[BC86] G. Brassard and C. Crepeau. Non-transitive transfer of confidence: a perfect zero-knowledge interactive protocol for SAT and beyond. In 27th Annual Symp. on Foundations of Computer Science (FOCS), pp. 188195, IEEE Computer Society Press, October 27-29 1986. Toronto, Ontario, Canada.

[BFM88] M. Blum, P. Feldman, and S. Micali. Non-interactive zero-knowledge and its applications. In Proceedings of the twentieth ACM Symp. Theory of Computing, STOC, pp. 103-112, May 2-4, 1988.

[BG84] M. Blum and S. Goldwasser. An efficient probabilistic public-key encryption scheme which hides all partial information. In Advances in Cryptology. Proc. of Crypto'84 (Lecture Notes in Computer Science 196), pp. 289-299, Springer-Verlag, New York, 1985. Santa Barbara, August 1984.

[Blu82] M. Blum. Coin flipping by telephone - a protocol for solving impossible problems. In digest of papers COMPCON82, pp. 133-137, IEEE Computer Society, February 1982. 
[Dep83] Department of Defense Trusted Computer System Evaluation Criteria. U.S. Department of Defense, August 15 1983. Also known as the Orange Book.

[Des] Y. Desmedt. Abuse-free cryptosystems: particularly subliminal-free authentication and signature. In preparation, available from author when finished.

[Des88] Y. Desmedt. Subliminal-free authentication and signature. May 1988. Presented at Eurocrypt'88, Davos, Switzerland, to appear in: Advances in Cryptology. Proc. of Eurocrypt 88 (Lecture Notes in Computer Science), Springer-Verlag.

[DGB87] Y. Desmedt, C. Goutier, and S. Bengio. Special uses and abuses of the Fiat-Shamir passport protocol. In C. Pomerance, editor, Advances in Cryptology, Proc. of Crypto'87 (Lecture Notes in Computer Science 293), pp. 21-39, Springer-Verlag, 1988. Santa Barbara, California, U.S.A., August 16-20.

[FFS87] U. Feige, A. Fiat, and A. Shamir. Zero knowledge proofs of identity. In Proceedings of the Nineteenth ACM Symp. Theory of Computing, STOC, pp. $210-217$, May 25-27, 1987.

[FS86] A. Fiat and A. Shamir. How to prove yourself: Practical solutions to identification and signature problems. In A. Odlyzko, editor, Advances in Cryptology, Proc. of Crypto'86 (Lecture Notes in Computer Science 263), pp. 186-194, Springer-Verlag, 1987. Santa Barbara, California, U. S. A., August 11-15.

[GMR] S. Goldwasser, S. Micali, and C. Rackoff. The knowledge complexity of interactive proof systems. to appear in Siam J. Comput., vol. 18, No. 1, January 1989.

[GMR88] S. Goldwasser, S. Micali, and R. Rivest. A digital signature scheme secure against adaptive chosen-message attacks. Siam J. Comput., 17(2), pp. 281-308, April 1988.

[GMW86] O. Goldreich, S. Micali, and A. Wigderson. How to prove all NP statements in zero-knowledge and a methodolgy of cryptographic protocol design. In A. Odlyzko, editor, Advances in Cryptology, Proc. of Crypto'8ô (Lecture Notes in Computer Science 263), pp. 171-185, Springer-Verlag, 1987. Santa Barbara, California, U. S. A., August 11-15.

[Lam73] B. W. Lampson. A note on the confinement problem. Comm. ACM, 16(10), pp. 613-615, October 1973. 
[PK79] G. J. Popek and C. S. Kline. Encryption and secure computer networks. ACM Computing Surveys, 11(4), pp. 335-356, December 1979.

[RS85] R. L. Rivest and A. Shamir. Efficient factoring based on partial information. In F. Pichler, editor, Advances in Cryptology. Proc. of Eurocrypt 85 (Lecture Notes in Computer Science 209), pp. 31-34, Springer-Verlag, Berlin, 1986.

[Sim83a] G. J. Simmons. Verification of treaty compliance-revisited. In Proc. of the 1983 IEEE Symposium on Security and Privacy, pp. 61-66, IEEE Computer Society Press, April 25-27 1983. Oakland, California.

[Sim83b] G. J. Simmons. The prisoners' problem and the subliminal channel. In D. Chaum, editor, Advances in Cryptology. Proc. of Crypto 83, pp. 51-67, Plenum Press N.Y., 1984. Santa Barbara, California, August 1983.

[Sim88] G. J. Simmons. How to insure that data acquired to verify treaty compliance are trustworthy. Proc. IEEE, 76(5), pp. 621-627, May 1988. 